\title{
Linewidth analysis of 40-GHz passively mode-locked multi-mode semiconductor lasers
}

\author{
Ramón Maldonado-Basilio, Sylwester Latkowski, Frederic Surre, Pascal
} Landais

\section{Research Institute for Networks and Communications Engineering (RINCE), Dublin City University, Glasnevin, Dublin 9, Ireland}

An experimental analysis on the quality of $40-\mathrm{GHz}$ radio-frequency signals generated by various passively mode-locked semiconductor lasers is addressed. The analysis is performed considering the frequency linewidth of $40-\mathrm{GHz}$ optically generated signals and the number of longitudinal modes selected by the cavity of each laser under study. Four of these devices are multi-quantum wells InAlGaAs Fabry-Pérot lasers. They have been engineered to exhibit a specific number of longitudinal modes: 4, 5, 10, and 22 modes, respectively. Another device under test is a bulk distributed Bragg reflector laser exhibiting 3 lasing modes. The last device under study is a quantum-dash Fabry-Pérot laser characterized by 40 lasing modes. From our experimental results it appears that, regardless the nature of the device cavity and active media, the strength of the passively mode-locked mechanism might be enhanced with the number of longitudinal modes oscillating into the laser cavity, allowing a reduction in the frequency linewidth. 


\section{Introduction}

Passively mode-locked (PML) semiconductor lasers have recently been investigated for pulse signal generation as they exhibit a periodic time variation of their output emission under dc-bias conditions. These devices allow for cheap generation of pulsed waveform signals at $40-\mathrm{GHz}$ or beyond as they do not require any direct or external modulation. There are several types of configurations for edge emitting PML lasers. Based on a gain-coupled distributed feedback (DFB) laser, 40-GHz self-modulation on these devices has been demonstrated along with their potential for all-optical clock-extraction [1,2]. These devices are composed of two sub-DFB sections merged to a phase section. Both sub-sections have a specific corrugation step and thus are spectrally detuned. The main drawback of these components is the complexity of their structure, which ultimately impacts their production cost. The distributed Bragg reflector (DBR) laser provides another solution. This is based on the large reflectivity spectrum of its Bragg mirror allowing the lasing of at least two main modes in the longitudinal spectrum. Its potential for all-optical clock recovery has been demonstrated at 40-GHz [3,4]. Pulse generation based on Fabry-Pérot (FP) cavities has also been investigated at $100-\mathrm{GHz}$ and $40-\mathrm{GHz}$. In the former case, a $408 \mathrm{~lm}$ long, multiquantum-well (MQW) la-ser was analyzed [5] whilst in the latter one, the study was carried out using InP-based quantum-dot lasers operating at the $1.55 \mathrm{~lm}$ window [6]. Recently, generation at 370-GHz and 1.1-THz has been demonstrated using MQW InAlGaAs FP lasers with a one-dimensional photonic band-gap embedded in its structure [7,8]. This photonic band-gap is achieved by etching shallow grooves on the p-side of the laser junction, perpendicular to the longitudinal axis of the device. The grooves are generating a variation of the local refractive index along the FP cavity. By placing the grooves at key positions, it is possible to control the longitudinal spectrum of the laser [9].

In some of the mentioned works, attention has been focused either on the frequency evolution in terms of the bias current or on the FWHM (full width at half maximum) linewidth of the radio-frequency (RF) generated signal. That analysis has been done considering a given number of longitudinal modes selected from the optical emission spectrum. In this work, the FWHM-linewidth of $40-\mathrm{GHz}$ optically generated signals is experimentally studied in terms of the number of longitudinal modes oscillating into a passively mode-locked semiconductor laser cavity. To this aim, we firstly consider four devices under test (DUT) whose structure is identical to that of the devices reported in $[7,8]$. These devices (labelled DUT-1 to DUT-4 throughout the text), however, have been engineered to exhibit a specific number of longitudinal modes by etching a given number of swallow grooves at key positions [9]. Since these four devices have been manufactured with the same structure and active layer material, we can expect the passively mode-locked mechanism being only affected by their optical spectrum and operational conditions. Secondly, we consider two more devices under test, an InGaAsP bulk DBR laser (DUT-5) and an InGaAsP/InAs quantum-dash FP laser (DUT-6). From our experimental results we show evidence that the quality of the passive mode-locking process, characterized in this work by the FWHM-linewidth of the RF-signal, can vary accordingly to the longitudinal 
spectrum of each laser under study. It is important to stress that we analyze the total number of modes oscillating into the laser cavity, without implementing any external optical processing such as a filtering of some longitudinal modes at the laser output, as it has been studied in $[10,11]$.

\section{Experiment}

The experimental setup is depicted in Fig. 1. The output of each DUT is coupled to a single mode fiber (SMF) and measured both electrically and optically. In the former case, the DUT collected out-put is injected into a $60-\mathrm{GHz}$ band-pass photo-detector (PD) and the RF-signal is measured by an electrical spectrum analyzer (ESA) set to $400 \mathrm{MHz}$ span and $10 \mathrm{kHz}$ video bandwidth. In the latter case, the DUT collected output is measured by a $20 \mathrm{MHz}$ resolution optical spectrum analyzer (OSA). An isolator (ISO) is placed at the DUT output to suppress back-reflections, whilst a 90/10 coupler is used to split the collected emission, allowing a simultaneous measurement in the electrical and optical domains.

Devices DUT-1 to DUT-4 are $1.05 \mathrm{~mm}$ long MQW InAlGaAs FP PML-lasers. They are single-section devices operating at dc-bias condition. They do not feature any saturable absorber in their cavity and their $2 \mathrm{~lm}$ wide ridge waveguide provides a spatial single mode output. In order to obtain a different number of longitudinal modes, these DUTs have been designed considering different numbers of grooves and groove patterns. The grooves are $1 \mathrm{~lm}$ deep and $2 \mathrm{~lm}$ wide. A scanning electron microscope (SEM) image of the wafer containing these analyzed DUTs is illustrated in Fig. 2, whilst the inset illustrates a close view of a groove etched at the laser top contact. These devices are not packaged and thus are tested by using a probe station. It is worth noticing that DUT-1 to DUT-4, as well as DUT-5 and DUT6 to be discussed below, have been analyzed using the same experimental setup depicted in Fig. 1, i.e. same isolator, fiber coupler, single mode fiber length and current supply. Thus, optical signals coming out from all the analyzed devices experience the same chromatic dispersion and the same carrier fluctuation induced by the power supply.

\section{Results}

Typical optical power collected in a single mode fiber versus bias current for the analyzed DUT-1 to DUT-4 MQW lasers are shown in Fig. 3. Devices are temperature controlled at 25 LC. The collected light was recorded on a slow response optical power meter as a function of the increasing and decreasing bias current $I_{\text {bias. }}$. All of these devices have a threshold current $\mathrm{I}_{\text {th }}$ around $27 \mathrm{~mA}$ and a maximum average collected output power of about $0.5 \mathrm{~mW}$ at 100 $\mathrm{mA}$. The number of grooves etched into the DUTs varies from 8 to 20 and have a minimal effect on the threshold current. This proves that the grooves implementation does not damage the active layer of our devices. The normalized collected spectra from de-vices DUT-1 to DUT-4 operating above the threshold current are shown in Fig. 4. Based on the properties of the FP cavity, devices DUT-1 to DUT-4 have a free-spectral range (FSR) of around 40-GHz, corresponding to $0.33 \mathrm{~nm}$ adjacent mode separation. 
From Fig. 4 it is observed that the optical spectrum envelope is not smooth and the longitudinal mode distribution is asymmetrical. Moreover, the number of main lasing modes associated with each DUT is not the same, and thus neither is their relative power and wavelength. All of this is so because of the quantity and separation of the etched grooves engineered into the p-side of the active layer devices. Here, we define the main lasing modes as those whose power is up to $20 \mathrm{~dB}$ (around 1\%) below the strongest longitudinal mode in the cavity. Thus, DUT-1 features two groups of dual-modes, centered at $1546.5 \mathrm{~nm}$ and 1551 $\mathrm{nm}$, respectively. DUT-2 has two-modes centered at $1544.8 \mathrm{~nm}$ and three-modes at 1547.2 $\mathrm{nm}$. Moreover, DUT-3 features two main groups of four-modes, separated by $2.9 \mathrm{~nm}$ and finally DUT-4 features a smoother FP-like spectrum. In all these spectra, the actual bias of each DUT is set to $65,70,90$, and $55 \mathrm{~mA}$, respectively. Thus, it is set a normalized ratio $1 / 2 \mathrm{I}_{\text {bias }}=\mathrm{I}_{\mathrm{th}} \mathrm{P} 1$ of 1.6, 1.3, 2.6 and 1.2, respectively. The bias is set to different levels in order to compensate for the different output powers collected at the photo-detector. This is so due to the differing alignment between the DUT output and the single mode fiber collecting the light.

The RF-signals generated by these devices under test are shown in Fig. 5. For the sake of clarity, this figure shows the relative out-put power for each RF-signal. In addition, their 40$\mathrm{GHz}$ frequency has been referenced to zero. From these experimental results, it is observed that the FWHM-linewidth of the RF-signal is quenched either as the number of longitudinal modes associated to each group is increased or as the wavelength separation between each multi-mode group is reduced. In particular, the FWHM-linewidth of the RF-signals generated by DUT-1 to DUT-3 is between 13 and $8.5 \mathrm{MHz}$, whilst it is around $700 \mathrm{kHz}$ for DUT-4.

The modulation of the output emission on multi-mode FP lasers originates from the nonlinear interactions between all the longitudinal modes co-existing in the laser cavity. In the case of a laser with uncorrelated modes, their phases are independent and the RF-signal exhibits a spectral linewidth related to the sum of the optical linewidths of all the longitudinal modes and the power distribution among them [3]. Conversely, for a passively mode-locked laser, the optical modes are equally spaced and their relative phases are fixed. This results in a reduction of the RF-signal line-width in comparison to that of a laser with uncorrelated modes [3]. Thus, the RF down-converted signal benefits from a phase correlation between all the lasing modes through the nonlinear intra-cavity effects, largely dominated by four-wavemixing (FWM) [8]. Indeed, fixed phase and beat-note linewidth reduction have been previously reported in [3,7] for three-mode and in [8] for two-mode PML-lasers, respectively. Considering the number of longitudinal modes selected by the FP-cavity only (i.e. without considering the power distribution among all the modes), a criterion to identify the passively mode-locking mechanism in PML-lasers is given by:

$$
\Delta v_{R F} \ll \sum_{i}^{M} \Delta v_{i} .
$$

In Eq. (1), $\Delta \mathrm{U}_{\mathrm{RF}}$ is the FWHM-linewidth of the RF-signal at the beat-ing (FSR) frequency, $\mathrm{M}$ is the total number of longitudinal modes and $\mathrm{Dm}_{\mathrm{i}}$ is the linewidth of the ith optical mode. The criterion above states that if the device is passively mode-locked, the FWHM-line-width of the RF-signal is smaller than the sum of the spectral line-widths corresponding to all the M 
main optical modes. It is important to mention that this criterion occurs after a series of measurements which assessed the origin of the passively mode-locking mechanism in multimode semiconductor lasers. The experimental and theoretical work in $[3,5,12]$ have demonstrated that the quenching of the FWHM-linewidth of the RF-signal reveals the passive mode-locking in this sort of devices. Indeed, the value of $\Sigma \Delta v_{i} / \Delta U_{R F}$ is also a good assessment of the mode-locking mechanism quality in these devices. Therefore, the results depicted in Fig. 5 show that DUT-1 to DUT-4 are passively mode-locked and the quality of the locking mechanism, illustrated by the value of the RF-signal linewidth, varies accordingly to their longitudinal spectrum The spectral linewidth reduction in terms of the number of longitudinal modes might be explained by considering the phase and amplitude noise due to the amplified spontaneous emission (ASE). Despite the fact that the phase of each longitudinal mode fluctuates with ASE, the reduced linewidth of the RF-signal in a PMLlaser implies that these phase fluctuations are largely synchronized. As the power of the longitudinal modes, or as the number of longitudinal modes is increased, the ASE level is reduced [3]. Therefore, the random phase fluctuations of the modes due to the ASE are decreased too. On the other hand, the longitudinal modes in our analyzed devices are separated $40-\mathrm{GHz}$, so then the dominant FWM effect is the carrier density modulation. It gives rise to the generation of side-bands nearby the adjacent modes, which behave as injection locking signals [3], transferring the phase of one mode to that of the adjacent mode and achieving mutual phase synchronization among modes. Therefore, it is expected that both number and power of longitudinal modes contribute to a reduction in the RF-signal spectral linewidth. Considering our experimental results, this explains why device DUT-4 exhibiting a FP-like optical spectrum and device QD-FP (discussed below) exhibiting a smoother spectrum, give rise to a RF-signal with a narrower FWHM-line-width in comparison to the other DUT-1 to DUT-3 analyzed devices.

Using the results illustrated in Figs. 4 and 5, the ratio between $\mathrm{Dm}_{\mathrm{i}}$ and $\mathrm{Dm}_{\mathrm{RF}}$ is depicted in Fig. 6 in terms of the number of optical modes, showing an exponentially increasing behavior. In this case, the linewidth $\mathrm{Dm}_{\mathrm{i}}$ of each longitudinal mode is around $100 \mathrm{-MHz}$ and it has been measured by using a heterodyning set-up at a resolution of $20 \mathrm{MHz}$. Thus, the minimum (maximum) ratio $\Sigma \Delta \mathrm{v}_{\mathrm{i}} / \Delta \mathrm{U}_{\mathrm{RF}}$ which is obtained for DUT-1 (DUT-4), is approximately 30 (3000). In the frame of reference of all-optical clock recovery, for instance, a ratio of such a value fulfils the minimum finesse re-choired for these applications. It is worth noticing that the frequency linewidth of the RF-signal can be further decreased using a DUT with powerful longitudinal modes, as it has been shown in [3,5]. This is due to the passive mode-locking mechanism being affected not only by the phase, but by the magnitude of all the lasing modes. This means that, in order to generate a RF-signal oscillating at 40$\mathrm{GHz}$ or at another specific frequency with an optimum line-width reduction, it can be possible to use a PML semiconductor la-ser with a powerful FP-like spectrum, as discussed below.

In order to compare our experimental results regarding DUT-1 to DUT-4, in Fig. 6 we have included two more experimental data which have been recorded by using the same experimental setup depicted in Fig. 1. DUT-5 is a PML DBR-laser whose main features are described in [4]. As it is depicted in Fig. 7, its longitudinal spectrum features three modes separated approximately by $0.3 \mathrm{~nm}$, which corresponds to a $39.7 \mathrm{GHz}$ free-spectral range. The resolved linewidths are 224.57 MHz, 74.133 MHz and 81.521 MHz, for the modes 1, 2, 
and 3, respectively. The side-mode-suppression ratio is approximately $17 \mathrm{~dB}$, confirming that this device is multi-mode. DUT-6 corresponds to a multi-mode Fabry-Pérot quantum-dash PML-laser. More information on this device is given in [11]. As it is depicted in Fig. 7, its optical spectrum envelope is certainly smoother and features around 40 main longitudinal modes when operating at $100 \mathrm{~mA}$ and temperature controlled at $25 \mathrm{LC}$. At these conditions, its collected power is around $1 \mathrm{~mW}$, the average line-width of each mode is $60-\mathrm{MHz}$ and the passively mode-locked frequency is approximately $39.78 \mathrm{GHz}$. The measured FWHMlinewidth of the RF-signal corresponding to DUT-5 (three-mode la-ser) and DUT-6 (40-mode laser) are around $24 \mathrm{MHz}$ and $25 \mathrm{kHz}$, respectively. It can be argued that these DBR and quantum-dash lasers have different active layer structures in comparison to those of our initially analyzed DUTs. Furthermore, the operating conditions of the quantum-dash FP are similar to those of DUT-1 to DUT-4, but it is not the case for the DBR. In particular, the ratio $\left[\mathrm{I}_{\text {bias }} / \mathrm{I}_{\mathrm{th}}-1\right]$ is 2.3 and 5 for the quantum-dash FP and DBR, respectively. However, the aim in overlapping these data in Fig. 6 is to illustrate that both the number and peak power of all lasing modes allocated in a pas-sively mode-locked multi-mode laser seem to be contributing to the linewidth quenching of the RF generated signal. Moreover, as it was previously discussed, the FWHM-linewidth of the RF-signal is a measure of the phase-locking mechanism strength exhibited by all the modes locked together in a semiconductor laser. Thus, it is possible that a laser characterized by a few but well-synchronized longitudinal modes might exhibit a very sharp frequency linewidth in comparison to a laser with many but poorly-synchronized optical modes. In order to disregard this ambiguity, our main devices under test (DUT-1 to DUT-4) have been engineered with a different number of longitudinal modes but manufactured by using the same substrate, active layer and waveguide structure. Thus, we can expect the passively mode-locked mechanism strength being the same into these four devices and only affected by their optical spectrum and operational conditions. Considering our experimental results (see Figs. 5 and 6), it appears the strength of the passively mode-locked mechanism might be enhanced with the number of longitudinal modes oscillating into the laser cavity, allowing a reduction in the frequency linewidth.

\section{Conclusion}

In this work we have experimentally analyzed the FWHM-line-width of the RF-signal generated by passively mode-locked semiconductor lasers. The analysis has been done in terms of the number of main longitudinal modes allocated into the laser cavity. The FWHMlinewidths of four MQW passively mode-locked FP semiconductor lasers have been compared to that of a DBR laser and a quantum-dash FP laser. Even though all of these devices are dc-biased, they generated a high quality $40-\mathrm{GHz}$ signal by a passive modelocking mechanism. In the present work we show evidence of a direct link between the FWHM-linewidth of the generated RF-signals and the number of main lasing modes selected by these lasers. 
Acknowledgment

This project is supported by Enterprise Ireland, Proof of Concept, Project Reference PC/2007/033. 


\section{References}

[1] M. Möhrle, B. Sartorius, C. Bornholdt, S. Bauer, O. Brox, A. Sigmund, R. Steingrüber, M. Radziunas, H.-J. Wünsche, IEEE J. Sel. Top. Quantum Electron. 7 (2) (2001) 217.

[2] W. Mao, Y. Li, M. Al-Mumin, G. Li, IEEE J. Light. Tech. 20 (9) (2002) 1705.

[3] J. Renaudier, G.-H. Duan, P. Landais, P. Gallion, IEEE J. Quantum Electron. 43 (2) (2007) 147.

[4] S. Latkowski, F. Surre, P. Landais, G.-H. Duan, Opt. Commun. 282 (2009) 2053.

[5] K. Sato, Electron. Lett. 37 (12) (2001) 763.

[6] G.-H. Duan, in: Proceedings of CLEO, San Jose, USA, paper CMG3, May 2008.

[7] S. Latkowski, F. Surre, P. Landais, Appl. Phys. Lett. 92 (8) (2008) 081109.

[8] S. Latkowski, F. Surre, R. Maldonado-Basilio, P. Landais, Appl. Phys. Lett. 93 (24) (2008) 241110.

[9] P. Landais, Patent No. S2005/0251, Ireland, 26 April 2005.

[10] K. Sato, IEEE J. Sel. Top. Quantum Electron. 9 (5) (2003) 1288.

[11] A. Shen, J.-G. Provost, A. Akrout, B. Rousseau, F. Lelarge, O. Legouezigou, F. Pommereau, F. Poingt, L. Legouezigou, G.-H. Duan, A. Ramdane, in: Proceedings of OFC'08, OThK1, 2008.

[12] G.-H. Duan, P. Landais, IEEE Photon. Tech. Lett. 7 (3) (1993) 278. 
Figures

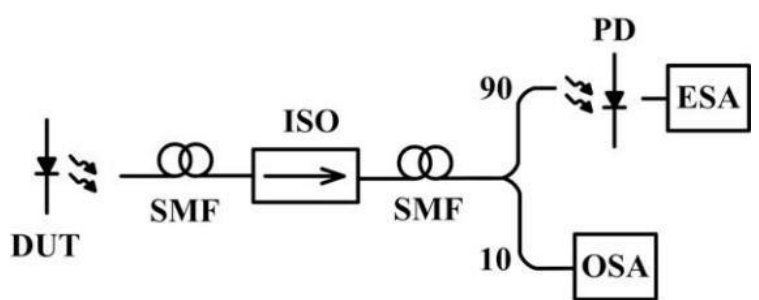

Fig. 1. Schematic of the experimental setup. 


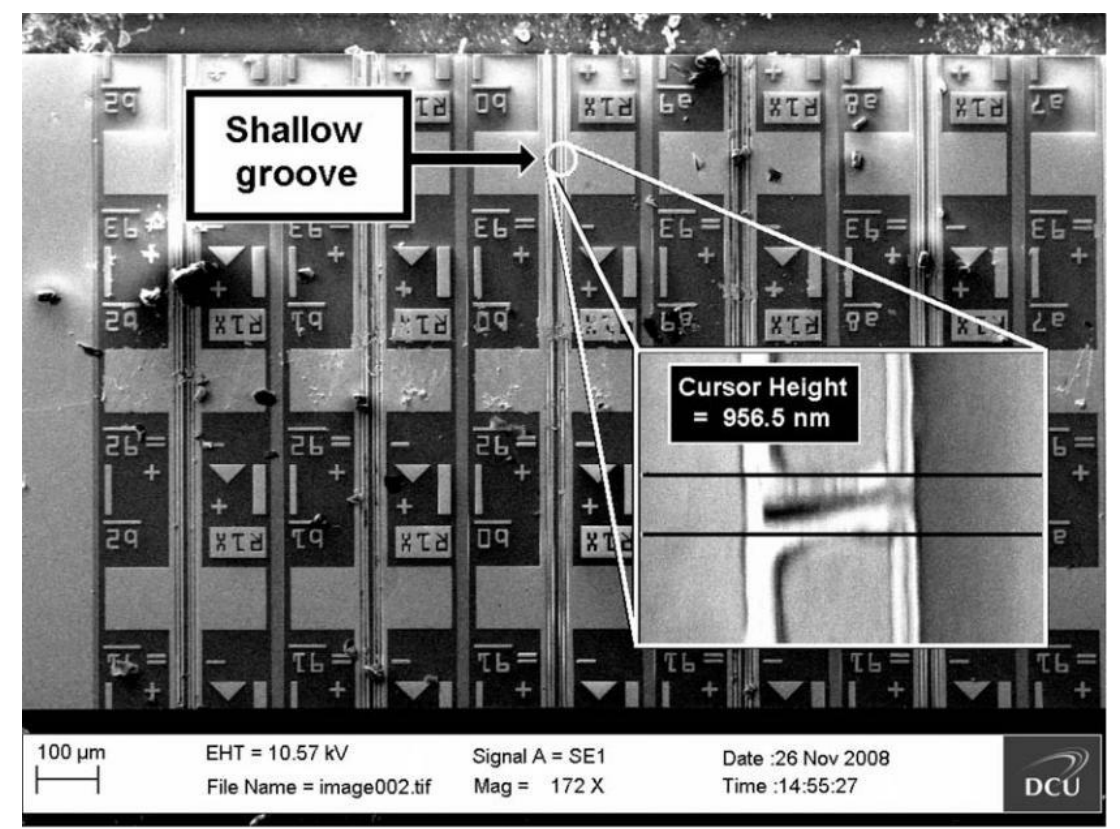

Fig. 2. SEM image of the wafer containing the analyzed DUT-1 to DUT-4 passively modelocked semiconductor lasers. The inset shows a close view of one groove at the top of a laser contact. 


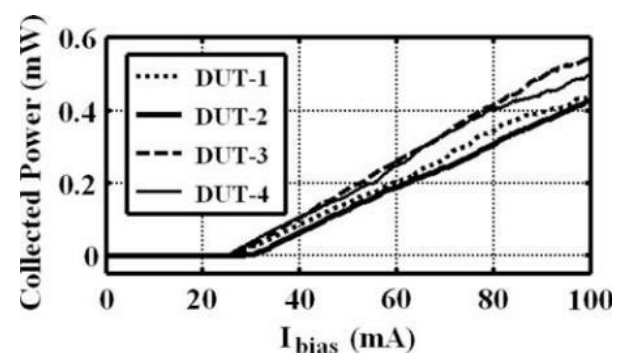

Fig. 3. Optical power collected in the single mode fiber as a function of the dc-bias current supplied to devices DUT-1 to DUT-4. 

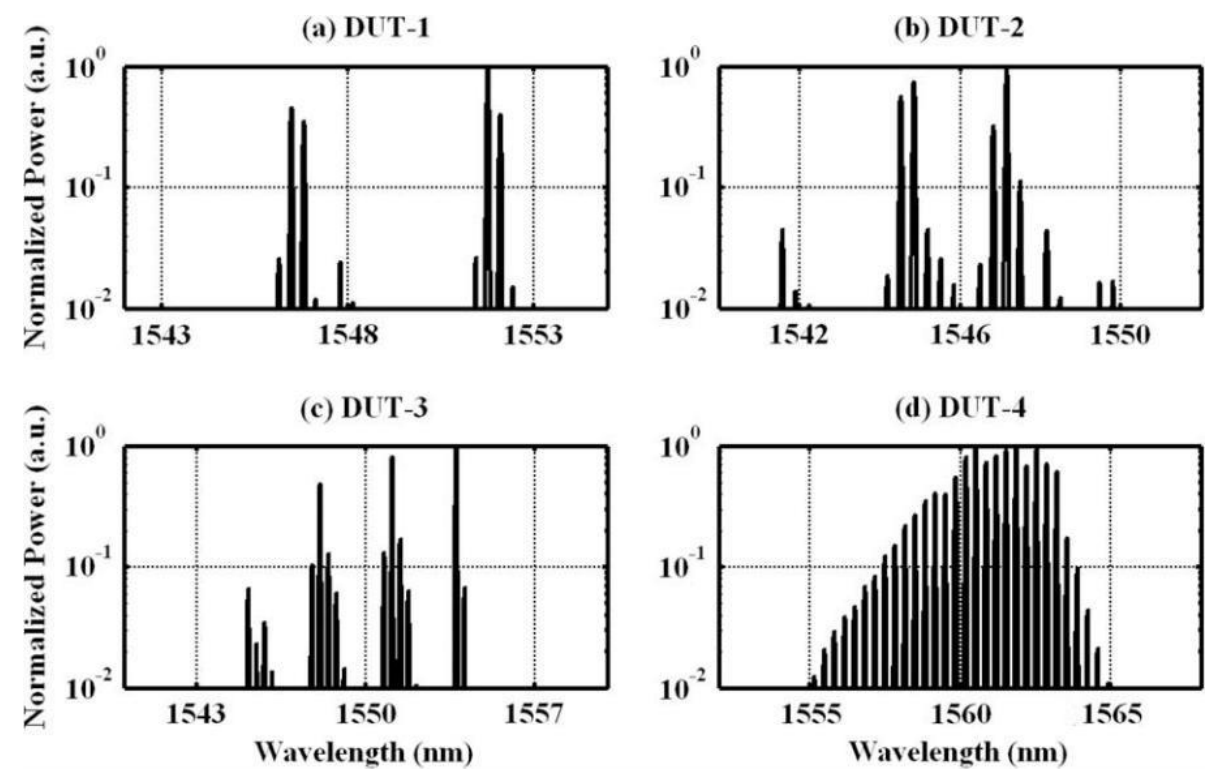

Fig. 4. Normalized optical spectra collected at the output of the analyzed devices DUT-1 (a) to DUT-4 (d) 


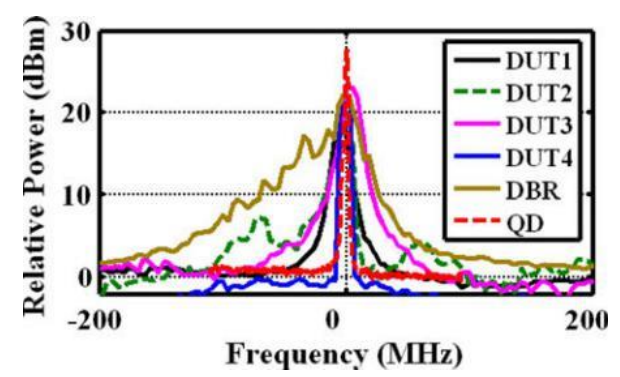

Fig. 5. RF-signals generated by the analyzed devices DUT-1 to DUT-4. The measured FWHM-linewidth for each tested device is as follows: $13 \mathrm{MHz}$ (DUT-1), 8.5 MHz (DUT-2), 8.5 MHz (DUT-3), 700 kHz (DUT-4), $24 \mathrm{MHz}$ (DBR), and $25 \mathrm{kHz}$ (QD). 


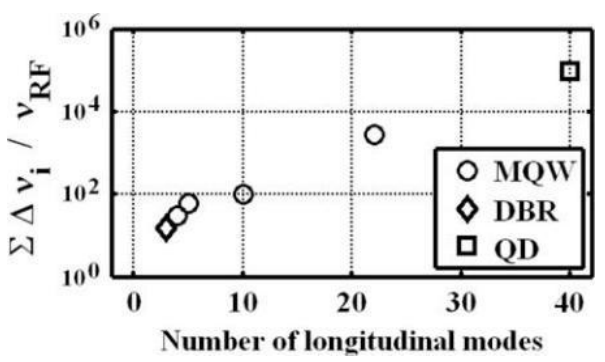

Fig. 6. Ratio ${ }^{\mathrm{P}} \mathrm{Dm}_{\mathrm{i}}=\mathrm{Dm} \mathrm{m}_{\mathrm{RF}}$ in terms of the number of longitudinal modes coexisting into the laser cavity. 

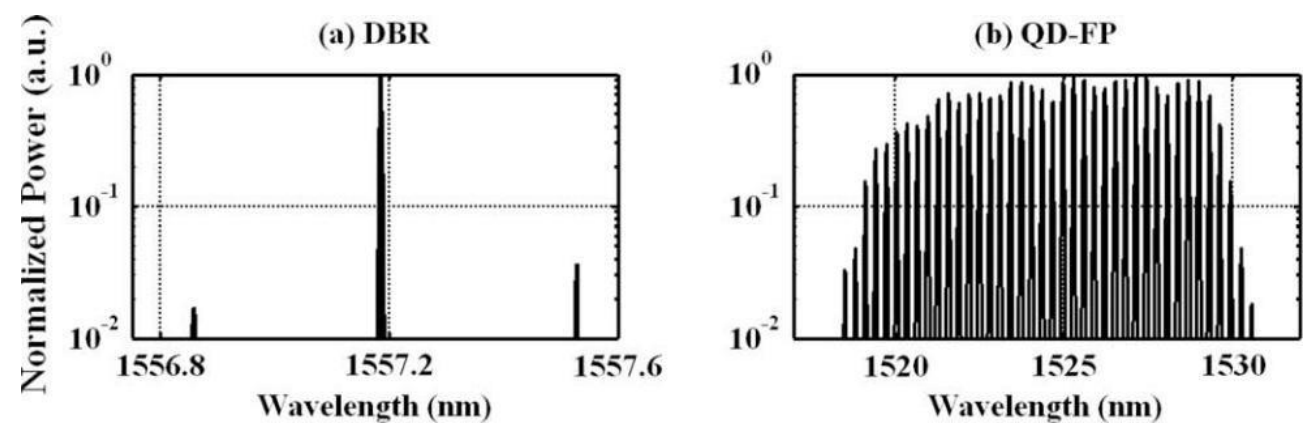

Fig. 7. Normalized spectra from a three-mode DBR (a) and a multi-mode quantum-dash Fabry-Pérot (b) lasers. 